\title{
66 Tubotympanic chronic suppurative otitis media (CSOM)
}

A A non-marginal perforation of the pars tensa is usually present.

B Middle ear polyps should be removed by the naked eye with a snare.

C Cholesterol granulomata may occur.

D Copious mucoid discharge is produced.

E Adenoidectomy may be indicated.

\section{Pathogenesis of acquired cholesteatoma}

A Congenital epidermoid rests may break through the outer attic wall.

B Squamous epithelium from the external meatus can migrate through a marginal perforation.

C Tumarkin's theory postulates an intratympanic negative middle ear pressure with collapse of the ear drum.

D Prolonged infection of the middle ear cleft may lead to squamous metaplasia.

E Bone erosion is mainly due to a pressure effect.

\section{In attico-antral CSOM}

A Deafness is always marked if ossicular damage has occurred.

B Copious malodorous otorrhoea is common.

C Otalgia is commonly a presenting symptom.

D A central perforation is invariable.

E Vertigo is due to blockage of the eustachian tube. 